\title{
On-line Support Vector Regression of the Transition Model for the Kalman Filter
}

\author{
Samuele Salti ${ }^{\mathrm{a}}$, Luigi Di Stefano ${ }^{\mathrm{a}}$ \\ ${ }^{a}$ Viale Risorgimento, 2 - 40135 Bologna, Italy
}

\begin{abstract}
Recursive Bayesian Estimation (RBE) is a widespread solution for visual tracking as well as for applications in other domains where a hidden state is estimated recursively from noisy measurements. From a practical point of view, deployment of RBE filters is limited by the assumption of complete knowledge on the process and measurement statistics. These missing tokens of information lead to an approximate or even uninformed assignment of filter parameters. Unfortunately, the use of the wrong transition or measurement model may lead to large estimation errors or to divergence, even when the otherwise optimal filter is deployed. In this paper on-line learning of the transition model via Support Vector Regression is proposed. The specialization of this general framework for linear/Gaussian filters, which we dub Support Vector Kalman (SVK), is then introduced and shown to outperform a standard, non adaptive Kalman filter as well as a widespread solution to cope with unknown transition models such as the Interacting Multiple Models (IMM) filter.

Keywords: Adaptive Transition Model, Visual Tracking, Support Vector Regression, Kalman filter, Interacting Multiple Models
\end{abstract}

Email addresses: samuele.salti@unibo.it (Samuele Salti), Preprint submitted to Image and Vision Computing 
The problem of hidden state estimation from noisy measurements is transversal to several disciplines, such as Statistic, Control Theory, Econometrics and, last but not least, Computer Vision. In Computer Vision, this problem usually emerges in the Filtering and Data Association [1] component of visual tracking algorithms, and it is typically dealt with by Recursive Bayesian Estimation (RBE) [2].

One of the main drawbacks to the deployment of this powerful and sound framework consists in the requirement to a priori specify a process dynamic, i.e. a transition model between states, that the user believes the system obeys. In many cases, this model is unknown and hence, empirically selected among a restricted set of standard ones. This approximate tuning of a recursive Bayesian filter may seriously degrade its performance, that could be optimal in case of correct system identification. Another solution to cope with the lack of knowledge on the transition model is to treat the system as a jump Markov system, i.e. a system that can follow different models at different time steps. Under this assumption, the user provides the RBE filter with a pool of possible models and let it choose the most likely one in each time frame, thereby having the filter partially tackling the uncertainty on the real system dynamics. Nevertheless, the choice of the models in the pool is left to the user and therefore, prone to error.

In this work we take a novel perspective to transition model identification: we propose to learn the transition model on-line by feeding the filter evolution to Support Vector Machines in regression mode (SVR). We provide first a conceptual solution suited to the general formulation of RBE and we discuss some issues related to every specific instantiation of this luigi.distefano@unibo.it (Luigi Di Stefano) 
general model. Then, we address the Gaussian/linear case and provide a detailed solution for the Kalman Filter, dubbed Support Vector Kalman (SVK) filter. Our choice is motivated by the state-of-the-art performance demonstrated by SVR [3] as well as, in case of Gaussian/linear systems, by the sound theoretical match between the assumptions on uncertainties underlying Kalman filtering and SVR. The SVK filter drastically reduces the number of parameters to be specified by the user, in particular the process noise covariance matrix, which is typically hard to estimate, but plays a significant role with respect to filter performance. Our algorithm also allows for obtaining a time-variant estimation of the transition model, and therefore results in a more adaptive filter than those usually proposed in previous literature. A preliminary version of our work was presented in [4].

\section{Recursive Bayesian Estimation}

The problem of hidden state estimation, $\mathbf{x}_{k} \in \mathbb{R}^{n}$, from noisy measures, $\mathbf{z}_{k} \in \mathbb{R}^{m}$, is typically solved in discrete-time systems via Recursive Bayesian Estimation. In this framework the system is completely specified by a first order Markov model compound of a law of evolution of the state, a measurement process and an initial state $\mathbf{x}_{0}$.

In a Bayesian approach, given the uncertainty affecting both the law of evolution of the state as well as the measurement process, represented by the noise Random Variables $\nu_{k} \in \mathbb{R}^{n}$ and $\eta_{k} \in \mathbb{R}^{m}$ affecting, respectively, the state evolution and the measurement process, the entities comprising the system are defined by PDFs, i.e. following the notation in [2],

- the transition model $\mathbf{x}_{k}=f_{k}\left(\mathbf{x}_{k-1}, \nu_{k}\right)$ is modeled by the PDF $p\left(\mathbf{x}_{k} \mid \mathbf{x}_{k-1}\right)$; 
- the observation likelihood $\mathbf{z}_{k}=h_{k}\left(\mathbf{x}_{k}, \eta_{k}\right)$ is modeled by the PDF $p\left(\mathbf{z}_{k} \mid \mathbf{x}_{k}\right)$

- the initial state is known via its $\operatorname{PDF} p\left(\mathbf{x}_{0}\right)$.

These PDFs are generally assumed to be known a priori and never updated.

Given this characterization of the target motion, a general but conceptual solution to the tracking problem can be obtained in two steps: prediction and update [2] (Fig. 2). In the prediction stage, the ChapmanKolmogorov equation is used to propagate the belief on the state at time $k-1$ to time $k$

$$
p\left(\mathbf{x}_{k} \mid \mathbf{z}_{1: k-1}\right)=\int p\left(\mathbf{x}_{k} \mid \mathbf{x}_{k-1}\right) p\left(\mathbf{x}_{k-1} \mid \mathbf{z}_{1: k-1}\right) d \mathbf{x}_{k-1} .
$$

where $\mathbf{z}_{1: k-1}$ is the set of all measurements up to frame $k-1,\left\{\mathbf{z}_{1}, \ldots, \mathbf{z}_{k-1}\right\}$. This usually corresponds to a spreading of the belief on the state, due to the increasing distance in time from the last measurement. In the update stage, the PDF is sharpened again by using the current measure $\mathbf{z}_{k}$ and the Bayes rule

$$
p\left(\mathbf{x}_{k} \mid \mathbf{z}_{1: k}\right) \propto p\left(\mathbf{z}_{k} \mid \mathbf{x}_{k}\right) p\left(\mathbf{x}_{k} \mid \mathbf{z}_{1: k-1}\right)
$$

This conceptual solution is analytically solvable only in a few cases. A notable one is when the law of evolution of the state and the measurement equations are linear and noises are Gaussian. In this situation, the optimal solution is provided by the Kalman filter [5]. The RBE framework for this case becomes:

$$
\begin{gathered}
\mathbf{x}_{k}=f_{k}\left(\mathbf{x}_{k-1}, \nu_{k}\right) \Rightarrow \mathbf{x}_{k}=\mathbf{F}_{k} \mathbf{x}_{k-1}+\nu_{k}, \mathbb{E}\left[\nu_{k} \nu_{k}^{T}\right]=\mathbf{Q}_{k} \\
\mathbf{z}_{k}=h_{k}\left(\mathbf{x}_{k}, \eta_{k}\right) \Rightarrow \mathbf{z}_{k}=\mathbf{H}_{k} \mathbf{x}_{k}+\eta_{k}, \mathbb{E}\left[\eta_{k} \eta_{k}^{T}\right]=\mathbf{R}_{k} .
\end{gathered}
$$




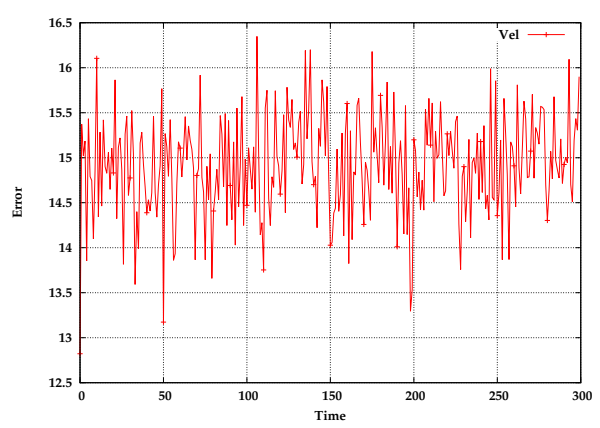

(a)

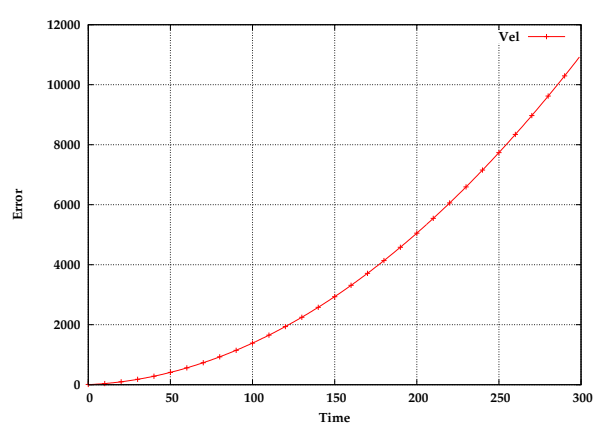

(b)

Figure 1: The impact of a wrong transition model: (a) the Kalman estimation exhibits, apart from the noise effect, a constant error (actual velocity - estimated velocity) if the wrong transition model is used; (b) the Kalman estimation diverges from the true velocity (i.e. the error increases) if a perturbed transition model is used (the noise effect is hardly perceivable here because of the much larger error scale).

and the mean and covariance matrix of the Gaussian posterior can be optimally estimated using the Kalman filter equations [5]. We will refer to $\mathbf{F}_{k} \in \mathbb{R}^{n \times n}$ and $\mathbf{Q}_{k} \in \mathbb{R}^{n \times n}$ as the transition matrix and the transition covariance, respectively.

\section{Motivation}

The difficulty of identifying a proper transition model for a specific application typically leads to empirical and suboptimal tuning of the filter parameters. The most widespread solutions to specify a transition model for tracking are to empirically select it among a restricted set of standard ones (such as constant position, i.e. Brownian motion, [6-8] or constant velocity [1, 9-11]) or learn it off-line from representative training data [12]. 
Besides the availability of these training sequences, which depends on the particular application, the major shortcoming of these solutions is that they do not allow to change the transition model through time, although this can be beneficial and neither the conceptual solution nor the solving algorithms require it to be fixed.

Approximate tuning of a recursive Bayesian filter may seriously degrade its performances, that could be optimal (e.g., when the assumptions of Kalman filtering are met) in case of correct system identification. In Fig. 1 we present two simple experiments to highlight the strong, detrimental impact of a wrongly identified transition model on an otherwise optimal and correctly tuned recursive Bayesian filter. In these simulations a point is moving along a line with constant acceleration and we try to estimate its position and velocity by two Kalman filters from measurements corrupted with Gaussian noise, whose constant covariance matrix is known and used as the measurement noise covariance matrix of the filters, $\mathbf{R}_{k}$. Hence, we are using the optimal estimator given the experimental setup. The only parameter that is wrongly tuned is the transition model, in particular, in one simulation we use a constant velocity transition matrix $\mathbf{F}_{k}$ instead of a constant acceleration one, whereas in the second simulation the transition model is a constant acceleration matrix whose parameters are perturbed by $5 \%$ of their value. The transition covariance matrix, $\mathbf{Q}_{k}$, was set very high, in order to compensate for the wrong transition matrix. Despite this, when using the wrong transition model the estimation exhibits a constant bias with respect to the real value while, when using the perturbed transition model the estimated and the true value of the velocity diverge. In other words, the estimation of an otherwise optimal estimator like the Kalman fil- 
ter can be arbitrarily wrong when an incorrect transition model is assumed. This highlights the main motivation behind our work.

\section{Related work}

Closely related to our work are the efforts devoted to the derivation of adaptive Kalman filters, that have been studied since the introduction of this filtering technique. In fact, our proposal can be seen as a new approach to build an adaptive Kalman filter. The main idea behind adaptive filtering schemes is that the basic source of uncertainty is due to the unknown noise covariances, and the proposed solution consists in estimating them on-line from observed data. One of the most comprehensive contributions in this field is provided in [13]. The paper reviews proposed approaches and classifies them according to four categories: Bayesian Estimation (BE), Maximum Likelihood Estimation (MLE), Correlation Methods (CM) and Covariance-Matching Techniques (CMT). Methods in the first category imply integration over a large dimensional space and can be solved only with special assumptions on the PDF of the noise parameters. MLE requires the solution of a non-linear equation that, in turns, is solvable only under the assumptions that the system is time invariant and completely observable and the filter has reached a steady state. Under these assumptions, however, only a time invariant estimation of the parameters of the noise PDF can be obtained. Correlation Methods, too, are applicable only to time invariant and completely observable systems. Finally, Covariance-Matching Techniques can estimate either process or measurement noise parameters and turn out to provide good and time-varying approximations for the measurement noise when the process noise is known. 
In [14], an improved correlation method is proposed, but the requirement on the stationarity of the system is not dropped. In [15], an approach able to simultaneously estimate both transition and covariance matrices, even if they are time-varying, is proposed. It is based on the construction of a recursive sequence of positive definite matrices representing the upper bounds for the real covariance matrices. Covariance matrices are then determined by minimizing such bounds, which turns out to require a timeconsuming convex optimization problem. In the context of visual tracking, [16] presents the application of an adaptive Kalman filter. The process and measurement errors are modified at every frame taking into account the degree of occlusion of the target: greater occlusion corresponding to greater value of measurement noise and vice versa, the two noises always summing up to one. In the extreme case of total occlusion, measurement noise is set to infinity and process noise to 0 . The method in [17] uses the term Adaptive to refer to an adaptive forgetting factor, that is used to trade off the contribution to the covariance estimate at the current time step of the covariance estimates for the previous time step and process noise. This is done to improve responsiveness of the filter in case of abrupt state changes.

Compared to all these proposals, our method makes no assumptions on the system. This allows our method to be more widely applicable and, in particular, to fit better the usual working conditions visual trackers have to deal with. Moreover, unlike BE, MLE and CM techniques, our proposal provides a time-varying noise statistics estimation. This is extremely important to allow the filter to dynamically weight the prediction on the state and the noisy measurement it has to fuse at each frame, e.g. to cope with occlusions when the measurement can be totally wrong and the prediction 
on the state is the only reliable source of information to keep on tracking the target. Unlike [16], our proposal is not specifically conceived for visual tracking and, hence, generally applicable. On the other hand, a general proposal like the one in [15], turns out to be less attractive for visual tracking than our filter, given the higher computational requirements. Finally, it is worth pointing out that, unlike all reviewed approaches, our proposal is adaptive in a broader sense, for it identifies on-line not only the process noise covariance matrix but also the transition matrix.

Another approach to deal with unknown covariance matrices and transition models is to rely on multiple standard models and let the filter choose the most adequate one in each time frame, that is effectively modeling it as Jump Markov system [2]. A celebrated algorithm based on this idea is the Interacting Multiple Model (IMM) filter [18], that has been used also for visual tracking $[19,20]$. We will show in the experimental results how our approach consistently outperforms IMM, having also the additional advantage to expose the user to less free parameters to be specified.

\section{SVMs in $\epsilon$-regression mode}

Our approach to transition model identification is based on Support Vector Machines [21] in $\epsilon$-regression mode (SVR) [3]. To make the paper self-contained, we provide a brief description of them.

To introduce SVMs as regressors, and in particular in $\epsilon$-regression mode, let us have a quick look at the regression of a linear model given a series of data $\left(\mathbf{x}_{i}, y_{i}\right)$. In $\epsilon$-regression mode the SVR tries to estimate a function of $\mathbf{x}$ that is far from training data $y_{i}$ at most $\epsilon$ and is at the same time as flat as possible. The requirement of flatness comes from the theory of complexity 
developed by [21] and ensures that we will get a solution with minimal complexity (hence, with better generalization abilities). In the linear case, the model we fit on the data is

$$
f(\mathbf{x})=\langle\mathbf{w}, \mathbf{x}\rangle+b
$$

and the solution with minimal complexity is given by the unique solution of the following convex optimization problem

$$
\begin{gathered}
\min \frac{1}{2} \| \mathbf{w}||^{2}+C \sum_{i=1}^{l}\left(\xi_{i}+\xi_{i}^{*}\right) \\
\left\{\begin{array}{l}
y_{i}-\left\langle\mathbf{w}, \mathbf{x}_{i}\right\rangle-b \leq \epsilon+\xi_{i} \\
y_{i}-\left\langle\mathbf{w}, \mathbf{x}_{i}\right\rangle-b \geq-\epsilon-\xi_{i}^{*}
\end{array}\right.
\end{gathered}
$$

The constant $C$ is a parameter and weights the deviations from the model greater than $\epsilon$. The problem is then usually solved using its dual form, that is easier both to solve and to extend so as to estimate also non-linear functions $([21])$.

\section{On-line transition model adaptation}

We propose to overcome the difficulties and shortcomings due to empirical tuning of the transition model by adapting it on-line.

In other terms, we propose to avoid to define $p\left(\mathbf{x}_{k} \mid \mathbf{x}_{k-1}\right)$, and instead use in its place a learned PDF $\tilde{p}\left(\mathbf{x}_{k} \mid \mathbf{x}_{k-1}\right)$, derived from a learned $\tilde{f}_{k}$.

Furthermore, we propose to learn the motion model using SVRs. A first reason to employ SVMs lays in their solid theoretical foundations, based on the statistical learning theory developed by Vapnik and Chervonenkis, which guarantee their ability to generalize from training data minimizing 
the over-fitting problem. Their use as regressors is probably less popular but even in this field they provide excellent performances [3]. In the case of Gaussian/linear systems, there is another important reason to use SVRs in combination with Kalman filters (the optimal RBE filter in such a case). The noise model assumed by the SVR is Gaussian, with mean and covariance being random variables whose distributions depend on two of its parameters, $C$ and $\epsilon$, as discussed in the very interesting work of Pontil et al. [22]. The mean, in particular, is uniformly distributed between $-\epsilon$ and $\epsilon$. Therefore, the SVR noise model is a superset of that assumed by the Kalman filter, i.e. a zero-mean Gaussian. In other words, the SVR is a theoretically sound regressor to apply in all the situations where the Kalman is the optimal filter.

In the context of RBE, given the first order Markovian assumption, the transition model represents the relationship between the state at time $k-1$ and that at time $k$. As we do not know the real states, to regress $f_{k}$ on-line we exploit the set of states predicted by the filter, $\hat{\mathbf{x}}_{1: k-1}$. In particular, we provide to the SVR as training data at time $k$ the tuples

$$
\left\langle\hat{\mathbf{x}}_{1}, \hat{\mathbf{x}}_{2}\right\rangle, \ldots,\left\langle\hat{\mathbf{x}}_{k-2}, \hat{\mathbf{x}}_{k-1}\right\rangle
$$

A global overview of the resulting system is provided in Fig. 2, whereas a detail algorithmic description is provided in Alg. 1.

Since the SVR can only regress functions $f: \mathbb{R}^{n} \rightarrow \mathbb{R}$, if the state vector has dimension $n, n$ SVRs are used, each one fed with tuples of the form $\left\langle\hat{\mathbf{x}}_{k-2}, \hat{\mathbf{x}}_{k-1}^{i}\right\rangle$, where the superscript $i$ denotes the $i$-th component of a vector.

Another important design choice is the use of a temporal window to select states for training. To use all the state transitions since the beginning 


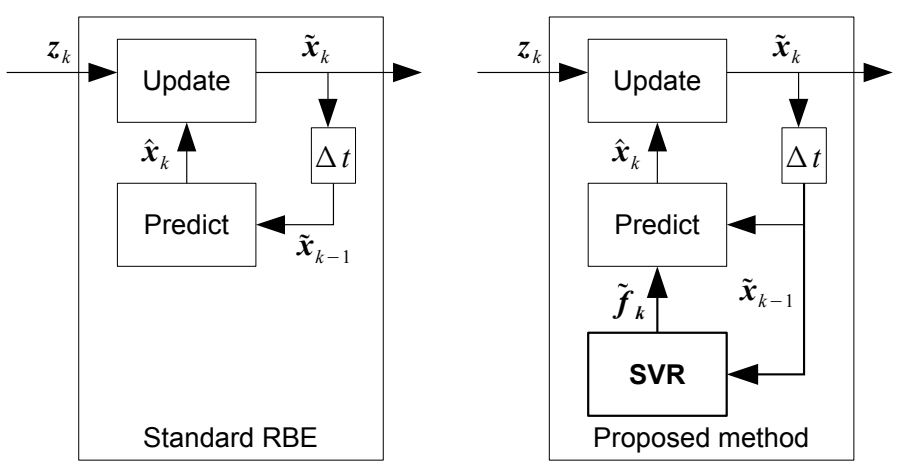

Figure 2: Global overview of a standard RBE filter (left) and of the proposed method (right).

of observations to learn the transition model for the current time slot can slow down the filter adaptation to sharp motion changes. A solution may be the use of dynamic SVR for time series regression, introduced in [23]. Though we believe that this may be beneficial, and can be an interesting investigation to carry on in the future, so far we have relied on a simpler solution, namely a sliding window of fixed length, so as to prevent too old samples from affecting the current estimate.

Finally, the influence of the time variable must be considered during regression. To understand this, consider the circular motion on the unit circle depicted in the leftmost chart of Fig.3. Assuming for clarity of the graphical explanation the state vector to consist of the $x$ position of the point only, some of the samples from which the SVR has to regress the transition model of this point are depicted in the second chart. As can be seen, without taking into account the evolution of the state through time, even with a perfect regression (represented by the dotted line in the second 

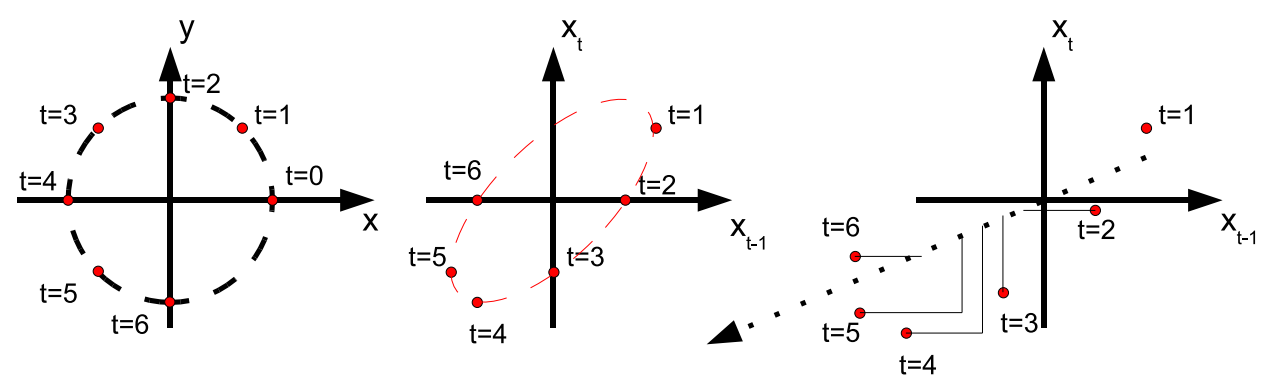

Figure 3: An example showing the importance of including time between the variables used for regression.

chart), it is impossible to have a correct prediction of the state at time $t$, given the state at time $t-1$ : for example, at time $t=4$ and $t=6$ the previous state, $x_{t-1}$, is equal for the two positions, but the output of the regression should be different, namely $x_{4}=-1$ and $x_{6}=0$. This situation can be disambiguated adding time as an input variable to the function to be regressed, as shown by the last chart.

To summarize, $n$ SVRs are used, where $n$ is the dimension of the state vector $\mathbf{x}_{k}$. The $i$-th SVR is trained at frame $k$ by using the following training set

$$
\left\{\left\langle k-2-W, \hat{\mathbf{x}}_{k-1-W}, \hat{\mathbf{x}}_{k-2-W}^{i}\right\rangle, \ldots,\left\langle k-1, \hat{\mathbf{x}}_{k-2}, \hat{\mathbf{x}}_{k-1}^{i}\right\rangle\right\}
$$

where $W$ is the length of the sliding window. We always use $W=10$ in our experiments.

In the following section we address in detail the linear-Gaussian case, when the Kalman filter is the optimal solution, and show how our framework can be instantiated to successfully and advantageously adapt the transition matrix and the associated noise covariance matrix on-line. 


\section{Support Vector Kalman}

In the case of linear process and measurement functions, of zero-mean Gaussian noise and Gaussian PDF for the initial state, all the subsequent PDFs of the state are (multivariate) Gaussians as well. Therefore, they are completely specified by their mean vector, that is usually also used as the state estimation, and their covariance matrix. The Kalman filter is the optimal estimator in this case.

Between the hypotheses of the Kalman filter there is linearity of $f_{k}$. Hence, three consequences immediately arise:

1. we must use a linear kernel, i.e. the SVR formulation introduced in Sec. 4;

2. we must modify it in order to regress a linear function;

3. we must discard the temporal variable.

In fact, the standard function learned by an SVR is (5), i.e. an affine mapping. As discussed by [24], a linear mapping can be learned without harming the general theory underneath SVM algorithms, since the linear kernel is a positive definite kernel. Moreover, a solving algorithm for the linear mapping was also proposed in the paper of [25], which introduced the standard and widespread solution for the affine case, i.e. the Sequential Minimal Optimization (SMO) algorithm.

As for the temporal variable, it would be beneficial even in the linear case to disambiguate situations similar to that depicted in Fig. 3. However, even if the evolution of the state is properly described by a linear law, its expansion along the temporal axis is not guaranteed in general to be linear.

Using this flavor of our framework, it is possible, given the training data 14 
in the considered temporal window, to obtain an estimate of $F_{k}$. Each vector of weights $\mathbf{w}_{k}^{i}$ regressed by the $i$-th SVR at time $k$ can be almost directly used as the $i$-th row of the estimated transition matrix, $\hat{F}_{k}$. The last but not least issue to be dealt with to deploy the SVR weights as rows of the Kalman transition matrix is normalization. Given the model regressed by the SVRs, it is also possible to associate an uncertainty to each predicted state variable, that can be used to define a temporal variant transition covariance matrix.

\subsection{Two-step normalization}

Typical implementations of SVMs require the input and output to be normalized within the range $[0,1]$ or $[-1,+1]$. While this normalization is a neutral preprocessing as far as the SVR output is concerned, it has subtle consequences when the weight vectors of the SVR are used within our proposal. To illustrate this, let us consider a simple example where a mapping from a scalar $p$ to a scalar $q$ is regressed, and the variables are normalized to the range $[-1,+1]$. Then

$$
\tilde{p}=\frac{2 p-p_{\max }-p_{\min }}{p_{\max }-p_{\min }}, \quad \tilde{q}=\frac{2 q-q_{\max }-q_{\min }}{q_{\max }-q_{\min }},
$$

where the superscript $\sim$ denotes the normalized variables and $p_{\max }, p_{\min }$ are the maximum and minimum value of the variable within the considered temporal window. Hence, the function of $p$ that gives the unnormalized $q$ is

$$
\begin{gathered}
\tilde{q}=w \tilde{p} \Rightarrow q=a p+b, a=\frac{2\left(q_{\max }-q_{\min }\right) w}{p_{\max }-p_{\min }} \\
b=q_{\max }+q_{\min }-\frac{\left(q_{\max }-q_{\min }\right)\left(p_{\max }+p_{\min }\right) w}{p_{\max }-p_{\min }}
\end{gathered}
$$


i.e., again an affine mapping. Therefore, using the unnormalized coefficient $a$ as an entry of the transition matrix $\hat{F}_{k}$ results in poor prediction, since the constant term is not taken into account. In order to obtain a linear mapping, that fits directly into the transition matrix of a Kalman filter, a two steps normalization must be carried out. Given a sequence of training data, a first normalization is applied,

$$
\bar{p}=p-\frac{p_{\max }+p_{\min }}{2}, \quad \bar{q}=q-\frac{q_{\max }+q_{\min }}{2} .
$$

These are the data on which the Kalman filter has to work. In other words, at every time step, the output of the previous time step must be renormalized if its value changes the minimum or maximum within the temporal window. This is equivalent to a translation of the origin of the state space and does not affect the Kalman filter itself. No normalization is required for the covariance matrix. After this normalization, the data can be scaled in the range $[-1,+1]$, as required by the SVR, according to

$$
\tilde{p}=\frac{2}{\bar{p}_{\max }-\bar{p}_{\min }} \bar{p}, \quad \tilde{q}=\frac{2}{\bar{q}_{\max }-\bar{q}_{\min }} \bar{q}
$$

where the subscripts have the same meaning as in (9). Using such a two steps normalization, the unnormalized function of the Kalman data is

$$
\tilde{q}=w \tilde{p} \Rightarrow \hat{q}=\frac{\left(\bar{q}_{\max }-\bar{q}_{\min }\right)}{\left(\bar{p}_{\max }-\bar{p}_{\min }\right)} w \bar{p},
$$

i.e. the required linear mapping.

\subsection{Adaptive transition covariance matrix}

As discussed in Sec. 3, the classical definition of an adaptive Kalman filter is more concerned with dynamic adjustment of $\mathbf{Q}_{k}$ than with the 
adaptation of the transition model $[14,17]$. Our proposal makes it easy to learn on-line the value of $\mathbf{F}_{k}$, but provides also an effective and efficient way to dynamically adjust the process noise. The value of $\mathbf{Q}_{k}$, in fact, is crucial for the performances of the Kalman filter. In particular, the ratio between the uncertainties on the transition model and on the measurements tunes the filter to be either more responsive but more sensitive to noise or smoother but with a greater latency in reacting to sharp changes in the dynamics of the observed system.

Within our framework, a probabilistic interpretation of the output of the SVR allows to dynamically quantify the degree of belief on the regressed transition model, and, consequently, the value of $\mathbf{Q}_{k}$. Some works have already addressed the probabilistic interpretation of the output of a SVR [26-28]. All of them estimate error bars on the prediction, i.e. the variance of the prediction. Therefore they are all suited to estimating the Gaussian covariance matrix of the regression output. We choose to use [28] since it is the simplest method and also turned out the most effective one in the comparison proposed in [28].

Given a training set, this method performs $k$-fold cross validation on it and considers the histogram of the residuals, i.e. the difference between the known function value at $\mathbf{x}_{i}$ and the value of the function regressed using only the training data not in the $\mathbf{x}_{i}$ fold. Then it fits a Gaussian or a Laplace PDF to the histogram, using a robust statistical test to select between the two PDFs. In our implementation, in accordance with the hypothesis of the Kalman filter, we avoid the test and always fit a Gaussian, i.e. we estimate the covariance as the mean squared residual. We also keep $\mathbf{Q}_{k}$ diagonal for simplicity. Hence, every SVR provides only the value of the 
diagonal entry of its row of $\mathbf{Q}_{k}$. As discussed before, however, learning from states is prone to degeneration of the learning loop into a filter unaffected by measurements. To avoid this, we prevent the covariance of every SVR to fall down a predetermined percentage of the corresponding entry of $\mathbf{R}$ (10\% in our implementation). This has experimentally proved to be effective enough to avoid the coalescence of the filter while at the same time preserving its ability to dynamically adapt the values of $\mathbf{Q}$.

Finally, such an estimation of the process noise covariance matrix allows for an intuitive interpretation of the $C$ parameter of the SVRs. Since $C$ weights the deviations from the regressed function greater than $\epsilon$, it is directly related with the smoothness of the Support Vector Kalman output. In fact, if $C$ is high, errors will be highly penalized, and the regressed function will tend to overfit the data, leading to greater residuals during the cross validation and to a bigger uncertainty on the transition model. This will result in a more noisy but more responsive output of the Kalman estimation. If, instead, $C$ is low, the SVR output will be smoother and the residuals during the cross validation will be smaller. The resulting tighter covariances will guide the Kalman filter towards smoother estimates of the state.

\section{Simulation results}

We provide first two simulations concerning a simple 1D estimation problem (i.e., a point moving along a line). In the first experiment, the motion is kept within the assumptions required by the Kalman filter, in particular there is a linear relationship between consecutive states. In the second one, a case of non-linear motion is considered. 

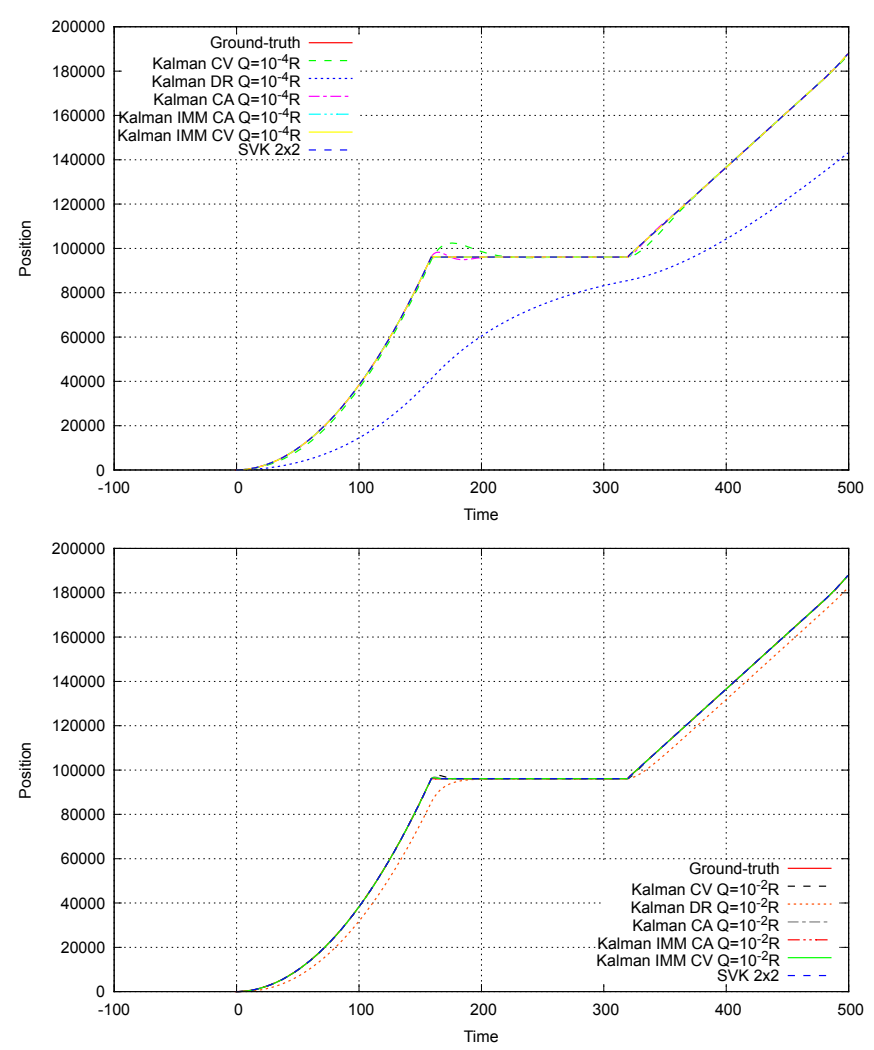

Figure 4: Charts showing the evolution of the filters against ground truth data in case of linear motion: the top one compares SVK to Kalman and IMM filters tuned for smoothness, the bottom one to Kalman and IMM filters tuned for responsiveness.

\subsection{Simulation of linear motion}

In both simulations, comparisons have been carried out versus three Kalman filters adopting different motion models, namely drift (Kalman DR), constant velocity (Kalman CV) and constant acceleration (Kalman CA), and two IMM filters, one using all the three previous models (IMM CA) and one using only drift and constant velocity (IMM CV). 


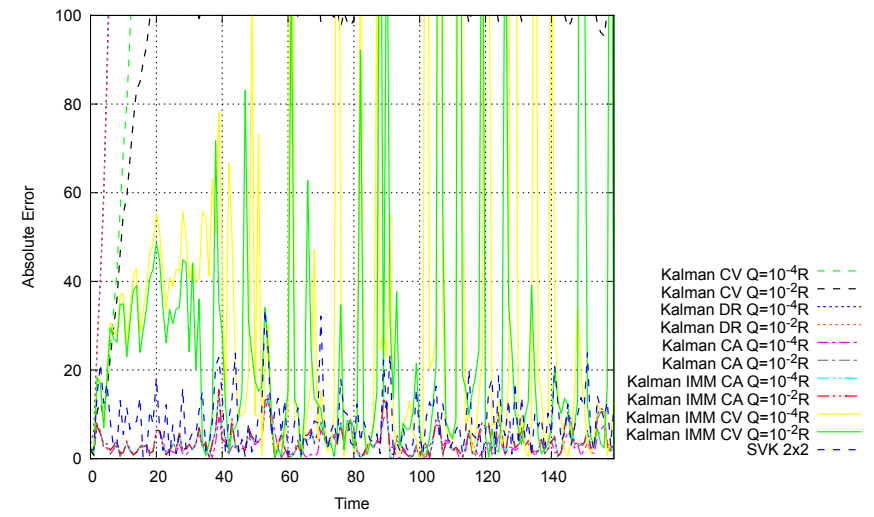

Figure 5: The chart reports absolute errors for the constant acceleration interval.

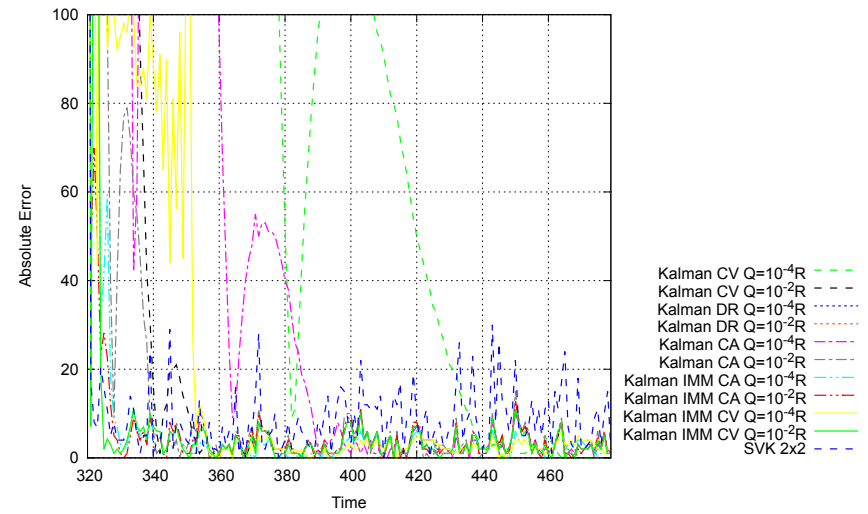

Figure 6: The chart reports absolute errors for the constant velocity interval. 
The model matrices are as follows:

$$
\mathbf{F}_{D R}=[1], \mathbf{F}_{C V}=\left[\begin{array}{cc}
1 & \Delta t \\
0 & 1
\end{array}\right], \mathbf{F}_{C A}=\left[\begin{array}{ccc}
1 & \Delta t & \frac{\Delta t^{2}}{2} \\
0 & 1 & \Delta t \\
0 & 0 & 1
\end{array}\right]
$$

Two different tunings were considered for each filter: a more responsive one, when $\mathbf{Q}$ has been set equal to $10^{-2} \mathbf{R}$; and a smoother one, with $\mathbf{Q}=10^{-4} \mathbf{R}$. For IMM filters, a uniform Transitional Probability Matrix [2] has been assumed, i.e. the filter has no preference in staying in the same regime with respect to jumping to a new one at each time frame. This has been done to allow the filter to best face the sharp motion changes present in this experiment, but it is worth noting that this is an unlikely tuning in a real-world scenario. Therefore, the performance of this filter can be overestimated by this experiment. Moreover, this is another example of the many degrees of freedom left to the user to tune with this kind of filters: a theoretical strength but also a practical obstacle and source of low performance due to wrong tuning. As far as SVK is concerned, it was fed with noisy measurements of the position and the velocity of the point, therefore regressing a $2 \times 2$ model matrix. The only rough tuning regards $C$, which is set equal to $2^{-10}$ in this simulation and to 2 in the non-linear case: intuitively, an easier sequence allows for deploying a smoother filter.

During the linear motion sequence, motion is switched every 160 samples between a constant acceleration, a constant position and a constant velocity law. Therefore, there is a time frame wherein the real motion of the point is exactly that described by the transition matrix of each Kalman filter. As for the IMM filters, the IMM CA filter has the perfect motion models pool to carry on estimation on this sequence, whereas the IMM CV has the 
optimal models only in two consecutive time frames. Results on the whole sequence are reported in Fig.4 and Tab.1. As for simulation parameters, $\mathbf{R}$ has been kept constant in time and equal to $100 * \mathbf{I}$, with $\mathbf{I}$ denoting the identity matrix; constant acceleration was $30.0 \mathrm{~m} / \mathrm{s}^{2}$, constant velocity was $1000 \mathrm{~m} / \mathrm{s}$ and $\Delta t$ was 0.5 . Gaussian noise with covariance matrix $\mathbf{R}$ was added to the data to produce noisy measurements for the filters.

As shown by the first column of Tab.1, the proposed SVK filter achieves the second best Root Mean Squared Error (RMSE) on the whole sequence. Its RMSE is very close to that of the optimal filter for this sequence, the IMM CA filter. This shows that on-line adaptation of the transition model can produce a very good estimate while requiring only a minimal set-up by the user. This is also shown by the two charts in Fig.4. At the scale of the charts, the estimations of SVK and of IMM filters are indistinguishable from the real state of the system, whereas the delay of Kalman DR and the overshots/undershots of Kalman CA and Kalman CV in presence of sharp changes of motion are clearly visible.

Going into more details, we separately analyze each of the three different parts of motion (Fig. 5, 7,6). Here, we discuss not only the performance on the whole interval associated with each motion law, but, also, those achieved in the final part of each interval (i.e., the last 80 samples). In fact, final samples allow to evaluate the accuracy at the steady state of the estimators, filtering out the impact of the delays due to the filter degree of responsiveness.

During the constant acceleration interval, Kalman CA and IMM CA perform best, both with the responsive as well as the smooth tuning. This is reasonable, since theoretically they are the optimal filters for this specific 
part of motion. Our filter, however, performs slightly worse than Kalman and IMM CA, but definitely better than Kalman and IMM CV and Kalman DR (2-nd column of tab.1). This is also demonstrated by Fig. 5, which, for better visualization, displays only absolute errors less than 50. Only our filter exhibits a trend similar to optimal ones. The poor performance of IMM CV is interesting: it shows that, when provided with an incorrect pool of models, i.e. quite a likely situation in a real deployment, IMM filters cannot achieve accurate state estimates. When considering only the steady state (5-th column of tab.1), the analysis does not change, partly because this interval is the very first one and, hence, there are no delays to recover, and partly because the Kalman and IMM CV and the Kalman DR do not have the proper transition matrix for this part and, thus, cannot recover from errors.

During the constant velocity part, the two responsive IMM filters and SVK have the best overall RMSE (3-rd column of tab.1). This is due to the delay accumulated by Kalman CV, theoretically the optimal filter, during the previous intervals. This highlights one of the major advantages brought in by SVK: in case of sharp changes of the motion law, dynamical update of parameters renders SVK even more accurate than the optimal filter due to its higher responsiveness. This is confirmed by Fig. 8, showing the position and the velocity variances estimated by SVK. It can be seen that, immediately after the change of motion law from constant position to constant velocity at sample 320, both variances significantly increase, somehow "detecting" such a change, thanks to the adaptive process noise modeling embodied into our filter. The resulting lower confidence in its predictions automatically turns the filter from smoothness to responsiveness, prevent- 


\begin{tabular}{cccccccc}
\hline \hline Filter & Whole & CA & CV & Drift & CA* & CV $^{*}$ & Drift* \\
\hline SVK 2x2 Model & 22.41 & 9.79 & 38.02 & 35.41 & 8.91 & 9.63 & 1.67 \\
Kalman IMM CA $Q=10^{-2} R$ & 18.23 & 4.62 & 31.62 & 3.61 & 4.50 & 4.22 & 4.62 \\
Kalman IMM CA $Q=10^{-4} R$ & 60.21 & 4.6 & 105.43 & 1.79 & 4.48 & 3.41 & 1.40 \\
Kalman IMM CV $Q=10^{-2} R$ & 56.03 & 76.91 & 36.78 & 44.82 & 102.86 & 4.37 & 4.36 \\
Kalman IMM CV $Q=10^{-4} R$ & 76.05 & 69.78 & 87.37 & 72.52 & 89.34 & 2.92 & 3.62 \\
Kalman CA $Q=10^{-2} R$ & 76.62 & 4.83 & 51.3 & 125.87 & 4.59 & 4.55 & 6.06 \\
Kalman CA $Q=10^{-4} R$ & 357.45 & 4.26 & 242.19 & 581.52 & 3.72 & 4.04 & 7.87 \\
Kalman CV $Q=10^{-2} R$ & 227.38 & 100.12 & 155.13 & 355.71 & 104.84 & 3.74 & 5.31 \\
Kalman CV $Q=10^{-4} R$ & 1680.37 & 1213.78 & 1160.73 & 2439.37 & 1416.30 & 49.82 & 109.30 \\
Kalman DR $Q=10^{-2} R$ & 4498.51 & 6015.22 & 4536.67 & 1793.30 & 8056.45 & 4757.75 & 2.77 \\
Kalman DR $Q=10^{-4} R$ & 29698.38 & 25771.38 & 31583.97 & 29279.53 & 35763.45 & 37809.42 & 16743.08 \\
\hline
\end{tabular}

Table 1: Comparison of RMSE on linear motion: first column reports the RMSEs on the whole sequence; then, partial RMSEs on each piece of motion are given as well as RMSEs concerning only the final part of each interval $($ marked with $*$ ), when the filter may have reached the steady state.

ing the overshots/undershots exhibited by standard Kalman filters. After a few samples, the covariance on the velocity decreases again, vouching that SVK has confidently learned the new model. Between IMM filters, only responsive ones perform well during this interval, due to the delay in reacting to the sharp change of smooth versions. Conversely, considering only the steady state (6-th column of tab.1), smooth filters are best, since they more effectively filter measurement noise. This is another strong indication of the importance of adapting the covariance matrix on-line to achieve good performance in every working condition. Considering only the steady state, Kalman CV exhibits, as expected, good performance. Unlike the CA interval, however, only the responsive tuning performs well since the smoother Kalman CV has accumulated too much delay to recover. This difference is 
due to the intrinsically higher smoothness of the CV model with respect to CA. Kalman CA, with both tunings, is another good performer during the steady state phase and this is also reasonable since a constant velocity motion may be seen as a special case of constant acceleration. Again, even in this phase SVK is by far closer to the optimal filters than to those adopting a wrong motion model and, visualizing only errors less than 50, it is the only one visible in Fig. 6, apart from those correctly tuned.

Finally, due to the delay accumulated by the other filters, SVK turns out the third best estimator in the constant position interval (4-th column of Tab.1). Only the optimally tuned IMM CA filters perform better. Note that, although IMM CV are optimally equipped for this interval, it comes immediately after the CA interval. Hence, the CV filters have to recover from accumulated delays and this results in a low global RMSE. Indeed,as far as the steady state is concerned, all the filters exhibit a good RMSE apart from very smooth ones, namely CV and DR tuned towards smoothness, since they do not recover from delays even after 80 samples. Unlike the other motion intervals, SVK keeps on being the best performer together with IMM $\mathrm{CA}$, even when only the steady state is considered. An explanation for this is provided again by the chart of covariances (Fig. 8). During the constant position part, the SVR is able to regress a very good transition matrix and both the uncertainties are kept really low compared to the values in $\mathbf{R}$. Therefore, the filter is highly smooth, as can be observed in the chart of absolute errors, and this keeps the RMSE low also in the last 80 frames.

Our proposal is robust to higher measurement noise, too. We report in Tab.2 the RMSEs for the same simulation, but with $\mathbf{R}=1000 \mathbf{I}$. Even in this case SVK turns out to be the overall second best thanks to its 


\begin{tabular}{cccccccc}
\hline \hline Filter & Whole & CA & Drift & CV & CA* & Drift* & CV $^{*}$ \\
\hline SVK 2x2 Model R=1000 & 43.36 & 31.35 & 9.33 & 67.93 & 28.29 & 5.23 & 30.56 \\
Kalman IMM CA $Q=10^{-2} R$ & 27.99 & 15.62 & 11.44 & 45.04 & 16.27 & 13.37 & 14.60 \\
Kalman IMM CA $Q=10^{-4} R$ & 111.47 & 15.78 & 5.66 & 195.15 & 16.57 & 17.27 & 10.47 \\
Kalman IMM CV $Q=10^{-2} R$ & 54.18 & 61.06 & 42.45 & 53.82 & 74.26 & 13.79 & 13.7 \\
Kalman IMM CV $Q=10^{-4} R$ & 116.60 & 105.94 & 28.87 & 173.47 & 105.36 & 19.17 & 83.12 \\
Kalman CA $Q=10^{-2} R$ & 79.65 & 15.36 & 130.17 & 52.94 & 14.52 & 19.17 & 14.3 \\
Kalman CA $Q=10^{-4} R$ & 357.69 & 13.33 & 581.70 & 242.46 & 11.75 & 17.28 & 10.94 \\
Kalman CV $Q=10^{-2} R$ & 228.08 & 100.97 & 356.26 & 156.61 & 106.77 & 16.81 & 11.71 \\
Kalman CV $Q=10^{-4} R$ & 1681.04 & 1214.90 & 2439.48 & 1162.36 & 1418.82 & 106.66 & 49.56 \\
Kalman DR $Q=10^{-2} R$ & 4500.00 & 6016.82 & 1793.01 & 4539.23 & 8059.09 & 8.78 & 4761.46 \\
Kalman DR $Q=10^{-4} R$ & 29699.11 & 25772.48 & 29279.76 & 31584.70 & 35764.94 & 16742.06 & 37810.78 \\
\hline
\end{tabular}

Table 2: Comparison of RMSE between different filters in case of higher measurement noise.

adaptive behavior. Considerations similar to previous ones apply to the three different parts of motion.

To summarize, simulations with linear motion laws show that the proposed SVR-based approach to on-line adaptation of the transition model is an effective solution for the tracking problem when the assumption of stationary transition matrix cannot hold due to the tracked system undergoing significant changes in its motion traits. They also show that trying to cope with the limited knowledge on the real transition model by deploying multiple approximate models with IMM filters is prone to obtain the same unsatisfactory performance when the right model is not present into the pool, beside requiring to guess a larger set of parameters, such as especially covariance matrices.

\subsection{Simulation of non-linear motion}

Given its ability to dynamically adapt the transition matrix, we expect SVK to be superior to a standard Kalman filter or to IMM filters in the 


\begin{tabular}{cccc}
\hline \hline R $=100$ & Whole & R=1000 & Whole \\
\hline SVK 2x2 Model & 20.61 & SVK 2x2 Model & 43.84 \\
Kalman IMM CA $Q=10^{-2} R$ & 38.53 & Kalman IMM CA $Q=10^{-2} R$ & 44.71 \\
Kalman IMM CA $Q=10^{-4} R$ & 48.88 & Kalman IMM CA $Q=10^{-4} R$ & 58.23 \\
Kalman IMM CV $Q=10^{-2} R$ & 289.90 & Kalman IMM CV $Q=10^{-2} R$ & 242.67 \\
Kalman IMM CV $Q=10^{-4} R$ & 236.11 & Kalman IMM CV $Q=10^{-4} R$ & 215.54 \\
Kalman CA $Q=10^{-2} R$ & 61.92 & Kalman CA $Q=10^{-2} R$ & 62.32 \\
Kalman CA $Q=10^{-4} R$ & 308.32 & Kalman CA $Q=10^{-4} R$ & 308.66 \\
Kalman CV $Q=10^{-2} R$ & 72.69 & Kalman CV $Q=10^{-2} R$ & 72.95 \\
Kalman CV $Q=10^{-4} R$ & 248.30 & Kalman CV $Q=10^{-4} R$ & 248.46 \\
Kalman DR $Q=10^{-2} R$ & 143.63 & Kalman DR $Q=10^{-2} R$ & 144.87 \\
Kalman DR $Q=10^{-4} R$ & 434.83 & Kalman DR $Q=10^{-4} R$ & 435.20 \\
\hline
\end{tabular}

Table 3: Comparison of RMSE in case of non-linear motion.

case of non-linear motion. In such a case, in fact, a time-varying linear function can approximate better than a fixed linear function the real nonlinear motion. Hence, to test this conjecture we have run simulations with a motion compound of two different sinusoidal parts linked by a constant position interval. The motion law of the two sinusoidal parts is as follows:

$$
\begin{array}{ll}
x_{1}(t)= & 300 t+300 \sin (2 \pi t)+300 \cos (2 \pi t), \\
x_{2}(t)= & 300 t-300 \sin (2 \pi t)-300 \cos (2 \pi t) .
\end{array}
$$

Aggregate results are shown in Fig. 9, Fig. 10 and Tab.3 for the same levels of measurement noise as in 7.1. In this case our filter proves to be the overall best, indeed confirming its superior flexibility.

\section{Experimental results}

In this section we provide experimental results on real data. The experiments concern 3D camera tracking and target tracking onto the image 
plane. For target tracker onto the image plane, we also provide quantitative results.

\subsection{D camera tracking}

In this experiment, we track the $3 \mathrm{D}$ position of a moving camera in order to augment the video content, taking as measurement the output of a standard camera pose estimation algorithm [29] fed with point correspondences established matching invariant local features, in particular SURF features [30]. Some snapshots are reported in Fig. 11. The snapshots show side-by-side the augmentation resulting from the use of Kalman CA (top image) and our SVK filter (bottom image). Both filters have been tuned to be as responsive as in 7.2 and measurement noise covariances has been adjusted to match the range of the input data. The sequence shows a fast change of motion of the camera, the purpose of filters being to keep the virtual object spatially aligned with the reference position, denoted for easier interpretation of results by a white sheet of paper. We can see that both filters exhibit a delay following the sharp motion change at frame 19, but SVK is subject to a much smaller translation error (e.g. frame 23), recovers much faster (SVK is again on the target by frame 27, Kalman CA only by frame 40) and, unlike Kalman CA, without any overshot (which Kalman CA exhibits from frames 27 to 40$)$.

\subsection{Tracking onto the image plane: qualitative evaluation}

In this experiments, we compare qualitatively our SVK to standard, non adaptive solutions for estimating an object trajectory in the image plane based on the mean-shift tracker introduced in [1]. We compare the 
original mean-shift (MS) tracker, the non-adaptive Kalman filter (KalmanMS tracker) and the IMM filter (IMM-MS tracker) to SVK. Kalman-MS, IMM-MS as well as SVK deploy the MS tracker as source of measurements. The MS tracker and the Kalman-MS tracker have been proposed in the original paper on mean-shift [1].

The basic MS tracker implicitly assumes a constant position transition model by letting the tracker start its search for the best position in each new frame exactly at the position where the target was found in the previous frame. Instead, the Kalman-MS tracker deployed in our experiment relies on a constant velocity motion model and the IMM-MS tracker has both a constant position and a constant velocity motion model.

Some snapshots of one test sequence are depicted in Fig. 12. The meanshift technique in general cannot withstand total occlusions, such as that shown in the third snapshot (Frame \# 069), because the MS tracker can be attracted by the background structure (e.g. the road in our experiment) if this turns out more similar to the target than the occluding object. For this reason the MS Tracker is unwilling to follow the object while it passes below the advertisement panel and stays in the last position where it could locate the target (frame \# 069 of Fig. 12). The Kalman-MS tracker and the IMM-MS tracker follow the previous dynamics of the target, thanks to the smoothness brought in by the transition models (frame \# 069 of Fig. 12). Nevertheless, since the way they weight the contribution of measurement and prediction on the state is fixed, they are finally caught back by the measurements (i.e. the MS tracker) continuously claiming the presence of the target in the old location, before the occluding object. Only the SVK is able to correctly guess the trajectory of the target while the latter is oc- 
cluded (frame \# 069 of Fig. 12) and continues to track it after the occlusion (frame \# 084 and subsequent frames of Fig. 12). This is due to the ability of the SVK to dynamically adjust the process noise covariance matrix, increasing its confidence on the motion of the object (i.e. to decrease the variance) while the object keeps moving with an approximatively constant motion law on the image plane (first part of the sequence, first two snapshots, from frame \# 001 to frame \# 051 of Fig. 12). Thanks to the high confidence gained on its motion model, the filter can reject the wrong measurements coming from the MS tracker during the occlusion. This happens again during the second occlusions at frame \# 201 of Fig. 12.

Similar considerations apply to the test sequence depicted in Fig. 13. Thanks to its adaptive behavior, SVK lets the mean shift tracker producing the new measurement at frame \# 86 start more on the left than the other two trackers, i.e. the filter has learned that the target is slowing down and exploits this adaptive knowledge to guide the measurements. This allows the mean shift tracker used by the SVK filter to recover the target as soon as it is not occluded (frame \# 101) and to stick to it during the subsequent frames (e.g. \# 127 and \# 195). In this sequence, which is less challenging than the previous one as the occlusion lasts less, the higher flexibility on the transition model of the IMM-MS tracker with respect to the simple Kalman-MS allows it to exhibit similar performance to that of the SVK.

\subsection{Tracking onto the image plane: quantitative evaluation}

To perform a quantitative comparison with non-adaptive methods, we used the methodology recently introduced in [31] and two sequences, Faceocc2 
and DavidIndoor, from a publicly available dataset ${ }^{1}$ introduced in two recent and very relevant proposals of adaptive appearance model trackers. We chose the methodology presented in [31] because it allows to compare methods independently of thresholds: it compares algorithms by simultaneously considering the mean Dice overlap and the Correct Track Ratio, i.e. the percentage of frames of a sequence that the tracker overlap is above a given threshold. This pair of values is plotted for every possible threshold value, resulting in curves like those depicted in Fig. 14: the higher the curve, the better the tracker performance. For more details on this methodology and the analyses that can be carried out on such curves, the reader is referred to $[31]$.

The SVK turns out the best in both sequences. Only on Faceocc2, the IMM filter offers similar performance. These sequences are designed to test appearance adaptive trackers. The mean-shift tracker, upon which all the evaluated trackers are based, is not adaptive: this is especially evident on the DavidIndoor sequence, where the target appearance between the first frame and the middle of the sequence changes drastically. This change of appearance makes all the trackers drift away from the target: the only one to remain on-target is the SVK, due to the high confidence gained on the target motion model, which overrules the wrong measures received from the Mean-shift tracker. In Faceocc2, where the greatest challenge is represented by partial occlusions, the mean-shift tracker is more effective. Nevertheless, trackers like IMM-MS and SVK, that offer time-variant transition models, obtain better performance than the basic algorithm. It is worth noting that our filter obtains the same performance of IMM-MS while requiring to set

\footnotetext{
${ }^{1}$ http://vision.ucsd.edu/ bbabenko/project_miltrack.shtml
} 
a lot less parameters.

\section{Parameters sensitivity}

To conclude the experimental validation of our proposal, we present a study about the sensitivity of the proposed algorithm to the parameter values. We used the DavidIndoor sequence presented in the previous section and consider the parameters $W$, i.e. the size of the sliding window, and $C$, i.e. the regularization term multiplier in the SVR objective function.

Results are reported in Fig. 15. We studied the effects of the parameters separately: in particular, we kept one parameter equal to the value used in the previous evaluation, and let the other vary. We report the mean Dice overlap over the whole sequence for the different parameter combinations. The proposed filter turns out robust to parameter tuning. In particular, the sliding window size is not a critical parameter, as the filter attains similar Dice overlaps even with small $W$, i.e. when it is provided with limited samples to regress a meaningful transition model. Similarly, the algorithm is not particularly sensitive to the value of $C$, as long as it is small enough to assign to the regressed transition model a low uncertainty, as explained in Sec. 6.2.

\section{Conclusions}

A new approach to build an adaptive recursive Bayesian estimation framework has been introduced, both from a theoretical point of view and in terms of its instantiation in the case of linear transition and measurement models and Gaussian noise. The proposed SVK filter has been shown to 
outperform a standard Kalman filter and an IMM filter while also requiring less parameters to be arbitrarily (and possibly wrongly) tuned.

It has also been highlighted how the proposed approach can be seen as a novel and general on-line adaptive Kalman filtering algorithm and thus beneficially deployable in a variety of hidden state estimation problems relying on different measurement sources, such as e.g. target tracking in the image plane based on color histograms and mean shift optimization as well as camera tracking based on interest points matching and camera pose estimation.

Endowing the vision of this work as a step towards a general and parameters free tracking system, an interesting future direction of research may deal with the insertion of algorithms for automatic on-line selection of SVR parameters. Finally, the instantiation of our proposal also in the case of non linear and non Gaussian tracking, in particular by modifying it in order to be beneficially used also with particle filters, would be a major contribution to foster its applicability and adoption.

\section{References}

[1] D. Comaniciu, V. Ramesh, P. Meer, Kernel-based object tracking, Transactions on Pattern Analysis and Machine Intelligence (PAMI) 25 (2003) 564-575.

[2] B. Ristic, S. Arulampalam, N. Gordon, Beyond the Kalman Filter: Particle Filters for Tracking Applications, Artech House, Boston, MA, USA, 2004.

[3] A. J. Smola, B. S. Olkopf, A tutorial on Support Vector Regression, Technical Report, Statistics and Computing, 1998.

[4] S. Salti, D. Luigi, On-line learning of the transition model for recursive bayesian estimation, in: Proc. of the 2nd International Workshop on Machine Learning for Vision-based Motion Analysis (MLVMA). 
[5] R. E. Kalman, A new approach to linear filtering and prediction problems, Transactions of the American Society of Mechanical Engineers (ASME)-Journal of Basic Engineering 82 (1960) 35-45.

[6] A. Adam, E. Rivlin, I. Shimshoni, Robust Fragments-based Tracking Using the Integral Histogram, in: Proc. of the Computer Society Conference on Computer Vision and Pattern Recognition (CVPR) - Volume 1, IEEE Computer Society Washington, DC, USA, 2006, pp. 798-805.

[7] S. Avidan, Ensemble tracking, in: Proc. of the International Conference on Computer Vision and Pattern Recognition (CVPR) - Volume 2, IEEE Computer Society Washington, DC, USA, 2005, pp. 494-501.

[8] R. T. Collins, Y. Liu, M. Leordeanu, Online Selection of Discriminative Tracking Features., Transactions on Pattern Analysis and Machine Intelligence (PAMI) 27 (2005) 1631-43.

[9] M. Harville, D. Li, Fast, integrated person tracking and activity recognition with plan-view templates from a single stereo camera, in: Proc. of the Computer Society Conference on Computer Vision and Pattern Recognition (CVPR) - Volume 2, IEEE Computer Society, Washington, DC, USA, 2004, pp. 398-405.

[10] M. Isard, J. MacCormick, BraMBLe: A bayesian multiple-blob tracker, in: Proc. of the International Conference on Computer Vision (ICCV) - Volume 2, IEEE Computer Society, Washington, DC, USA, 2001, pp. 34-41.

[11] I. Haritaoglu, D. Harwood, L. S. Davis, W4: Real-time surveillance of people and their activities, Transactions on Pattern Analysis and Machine Intelligence (PAMI) 22 (2000) 809-830.

[12] P. Prez, C. Hue, J. Vermaak, M. Gangnet, Proc. of the color-based probabilistic tracking, in: Proceedings of the Seventh European Conference on Computer Vision (ECCV) - Part I, Lecture Notes in Computer Science (LNCS), Springer-Verlag, London, 2002, pp. 661-675.

[13] R. Mehra, Approaches to adaptive filtering, Transactions on Automatic Control 17 (1972) 693-698.

[14] M. Oussalah, J. De Schutter, Adaptive Kalman filter for noise identification, in: Proc. of the 25th International Conference on Noise and Vibration Engineering 
(ISMA), Katholieke Universiteit, Leuven, Belgium, 2000, pp. 1225-1232.

[15] Y. Liang, D. X. An, D. H. Zhou, Q. Pan, A finite-horizon adaptive Kalman filter for linear systems with unknown disturbances, Signal Processing 84 (2004) 2175-2194.

[16] S.-K. Weng, C.-M. Kuo, S.-K. Tu, Video object tracking using adaptive Kalman filter, Journal of Visual Communication and Image Representation 17 (2006) 11901208.

[17] Y. Zhang, H. Hu, H. Zhou, Study on adaptive Kalman filtering algorithms in human movement tracking, in: Proc. of the IEEE International Conference on Information Acquisition (ICIA), pp. 11-15.

[18] E. Mazor, A. Averbuch, Y. Bar-Shalom, J. Dayan, Interacting multiple model methods in target tracking: a survey, IEEE Transactions on Aerospace and Electronic Systems 34 (1998) 103-123.

[19] M. E. Farmer, R.-L. Hsu, A. K. Jain, Interacting multiple model (IMM) Kalman filters for robust high speed human motion tracking, in: International Conference on Pattern Recognition, volume 2, IEEE, 2002, pp. 20-23.

[20] J. Burlet, O. Aycard, A. Spalanzani, C. Laugier, Pedestrian tracking in car parks: an adaptive interacting multiple models based filtering method, in: Intelligent Transportation Systems Conference, IEEE, 2006, pp. 462-467.

[21] V. N. Vapnik, The Nature of Statistical Learning Theory, Springer-Verlag, New York, NY, USA, 1995.

[22] M. Pontil, S. Murkerjee, F. Girosi, On the Noise Model of Support Vector Machine Regression, Technical Report, Massachusetts Institute of Technology, Cambridge, MA, USA, 1998.

[23] L. Cao, Q. Gu, Dynamic Support Vector Machines for non-stationary time series forecasting, Intelligent Data Analysis 6 (2002) 67-83.

[24] T. Poggio, S. Mukherjee, R. Rifkin, A. Rakhlin, A. Verri, b, Technical Report CBCL Paper 198/AI Memo 2001-011, Massachusetts Insititute of Technology, Artificial Intelligence Laboratory, 2001.

[25] J. C. Platt, Fast Training of Support Vector Machines Using Sequential Minimal Optimization, MIT Press, MA, USA, Cambridge, MA, USA, pp. 185-208.

[26] J. Gao, S. Gunn, C. Harris, M. Brown, A probabilistic framework for SVM regression 
and error bar estimation, Machine Learning 46 (2 January 2002) 71-89.

[27] W. Chu, S. Keerthi, C. J. Ong, Bayesian Support Vector Regression using a unified loss function, Transactions on Neural Networks 15 (2004) 29-44.

[28] C.-J. Lin, R. C. Weng, Simple Probabilistic Predictions for Support Vector Regression, Technical Report, Department of Computer Science, National Taiwan University, 2004.

[29] G. Schweighofer, A. Pinz, Robust pose estimation from a planar target, Transactions on Pattern Analysis and Machine Intelligence (PAMI) 28 (2006) 2024-2030.

[30] H. Bay, A. Ess, T. Tuytelaars, L. J. V. Gool, Speeded-Up Robust Features (SURF), Computer Vision and Image Understanding 110 (2008) 346-359.

[31] S. Salti, A. Cavallaro, L. Di Stefano, Adaptive Appearance Modeling for Video Tracking: Survey and Evaluation, Trans. on Image Processing (2012 (to appear)). 


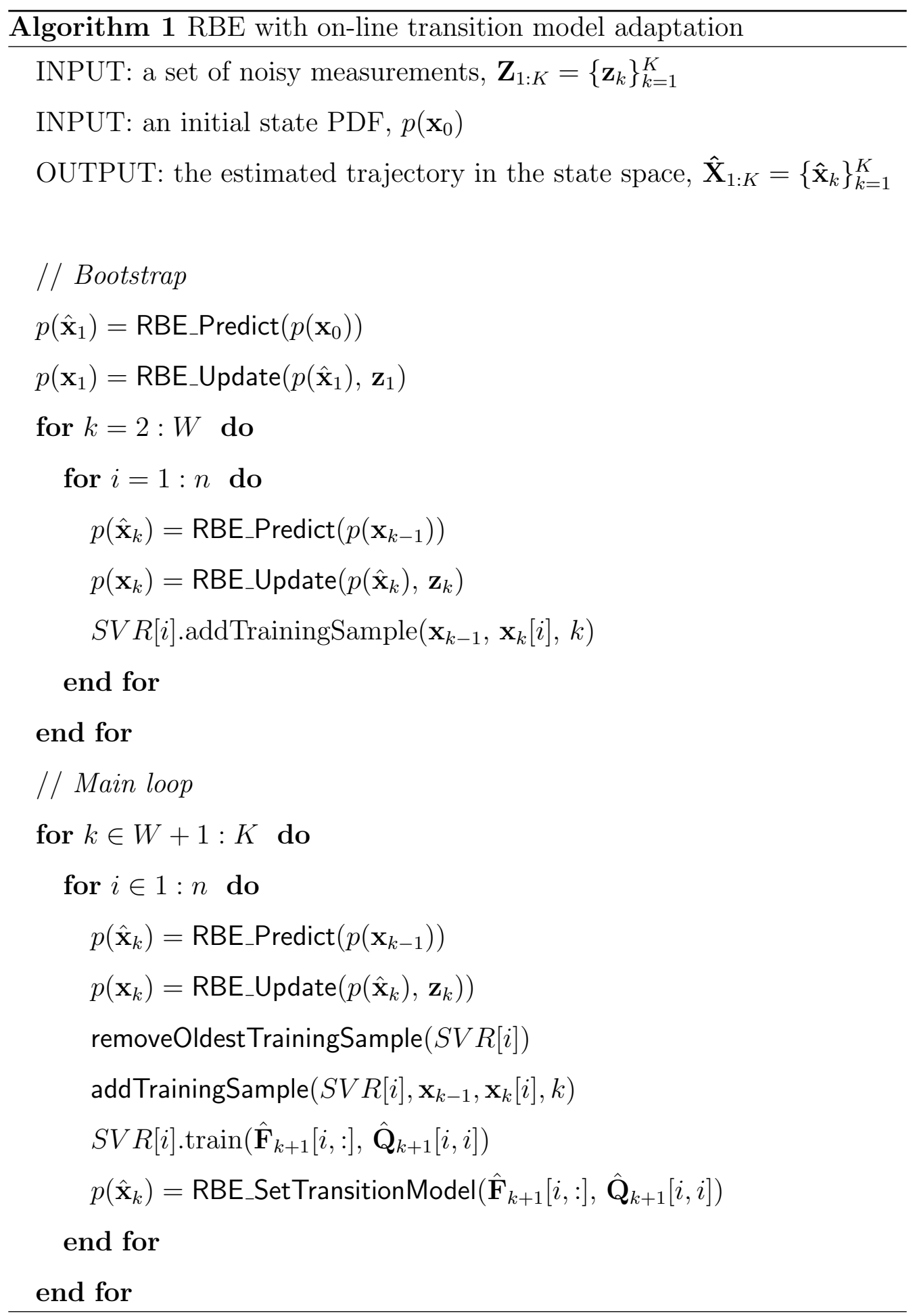




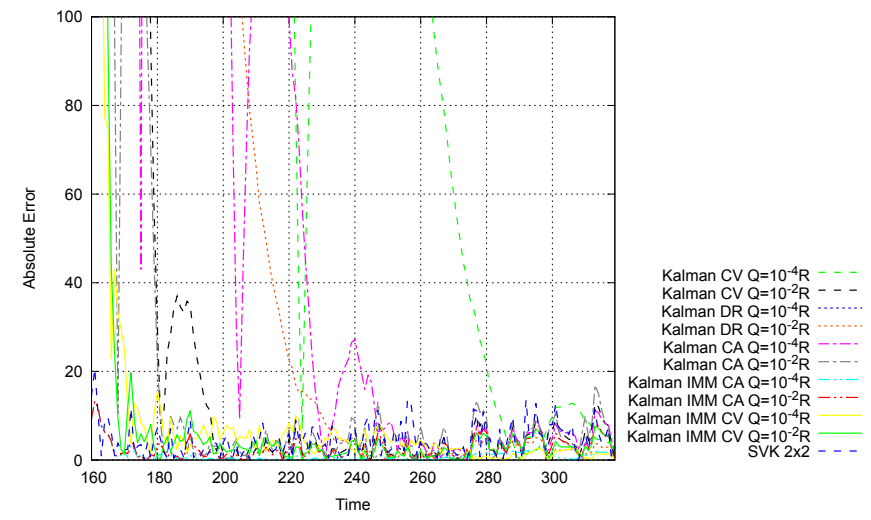

Figure 7: The chart reports absolute errors for the constant position interval.

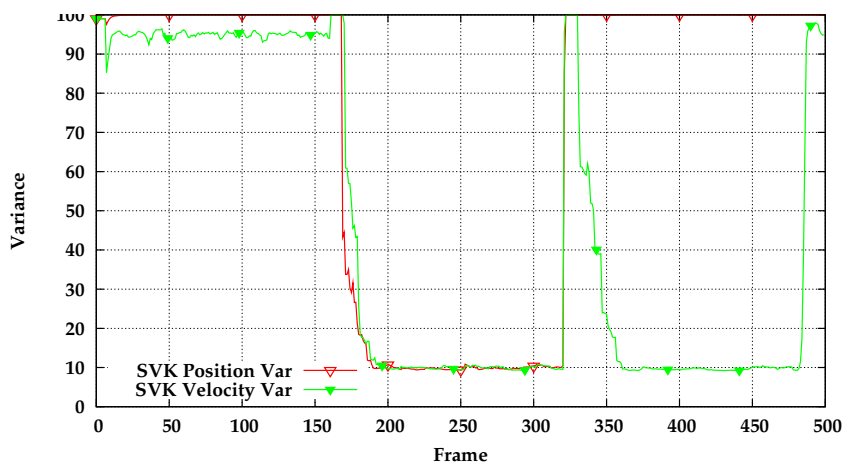

Figure 8: The chart shows the covariances on state variables provided by SVK throughout the whole sequence. 

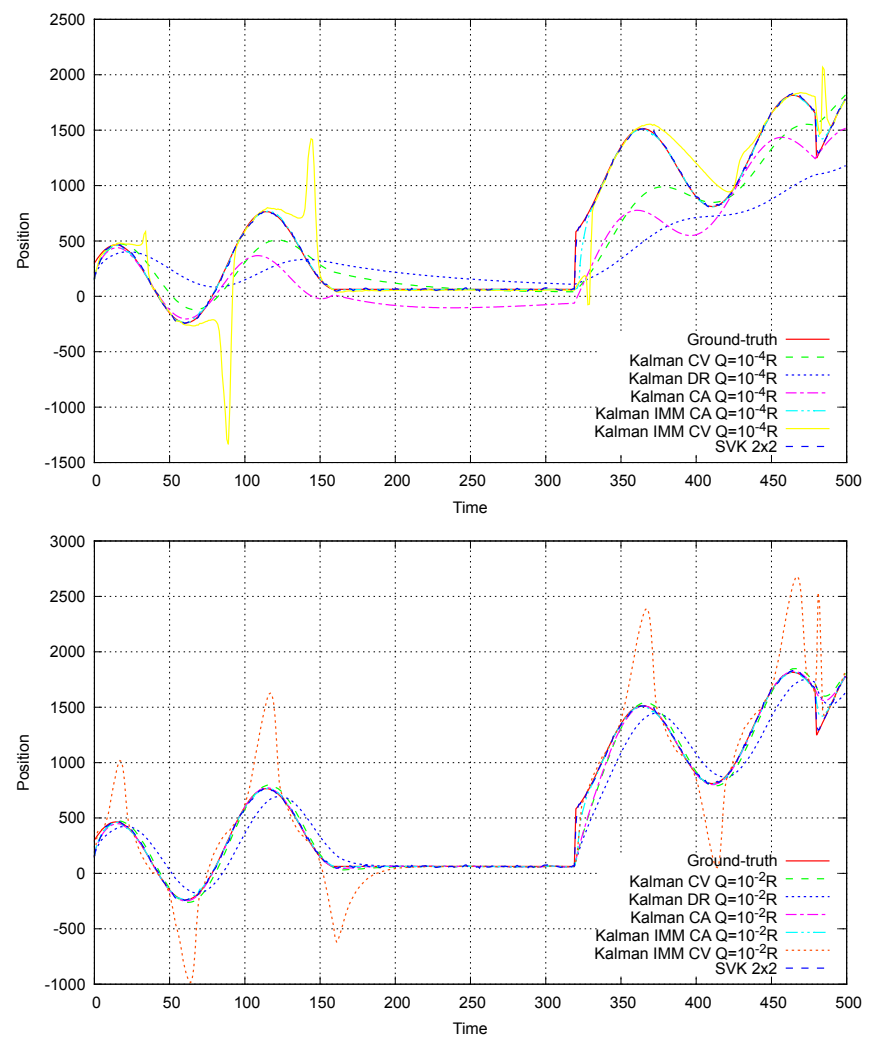

Figure 9: Simulation dealing with non-linear motion with $\mathbf{R}=100 \mathbf{I}$. Chart on top compares SVK to Kalman filters tuned for smoothness, the bottom one to Kalman filters tuned for responsiveness. At this scale, the estimation of our filter is almost indistinguishable from the ground truth. 

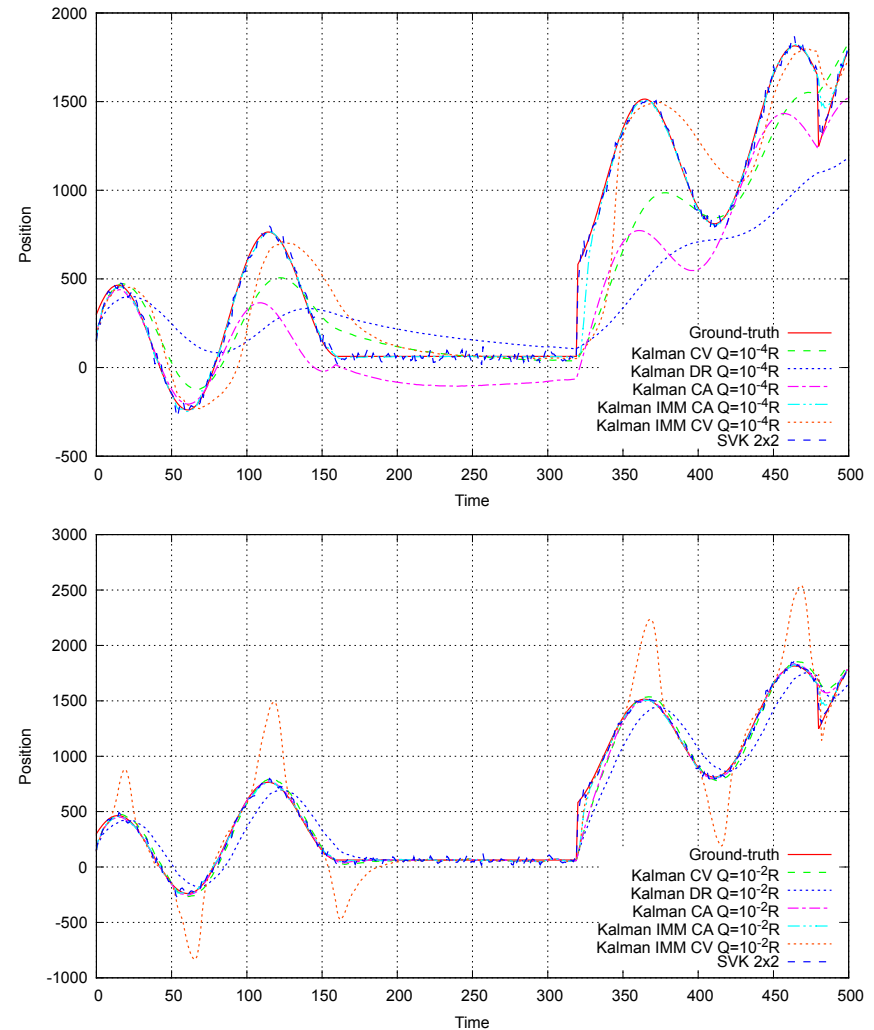

Figure 10: Simulation dealing with non-linear motion with $\mathbf{R}=1000 \mathbf{I}$. Chart on top compares SVK to Kalman filters tuned for smoothness, the bottom one to Kalman filters tuned for responsiveness. 

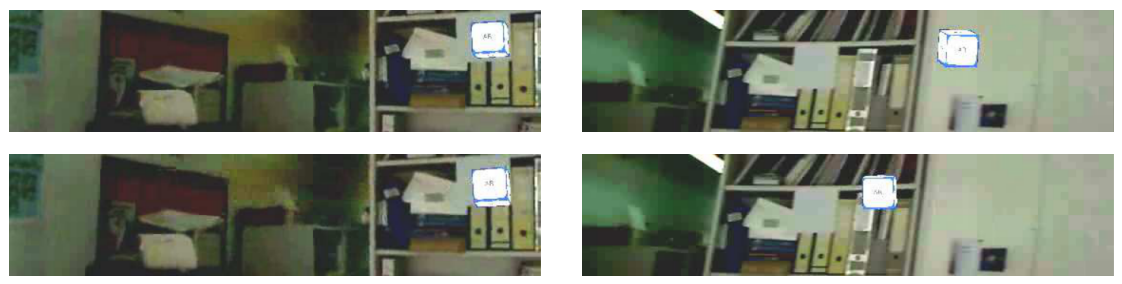

(a) 17 cropped

(b) 21 cropped
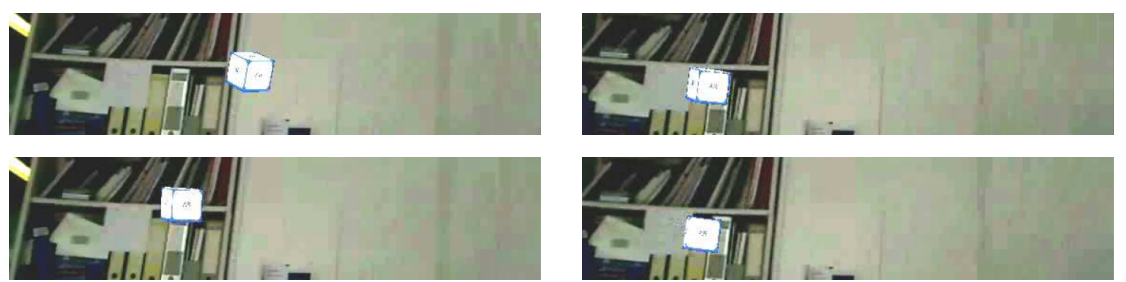

(c) 23 cropped

(d) 25 cropped
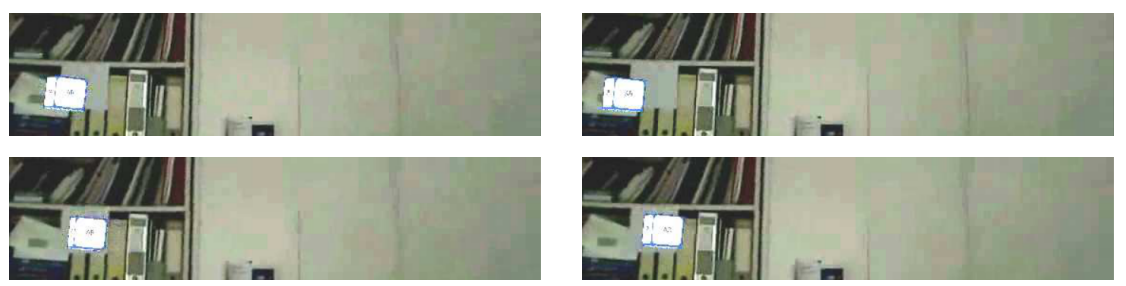

(e) 27 cropped

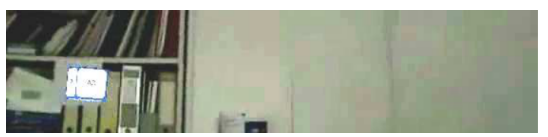

(f) 28 cropped
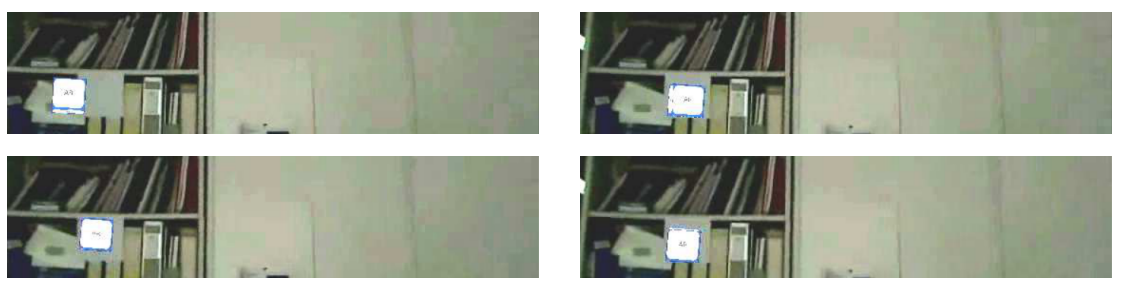

(g) 34 cropped

(h) 40 cropped

Figure 11: Some of the most significant frames (top: Kalman CA, bottom: SVK) from the 3D camera tracking experiment. 


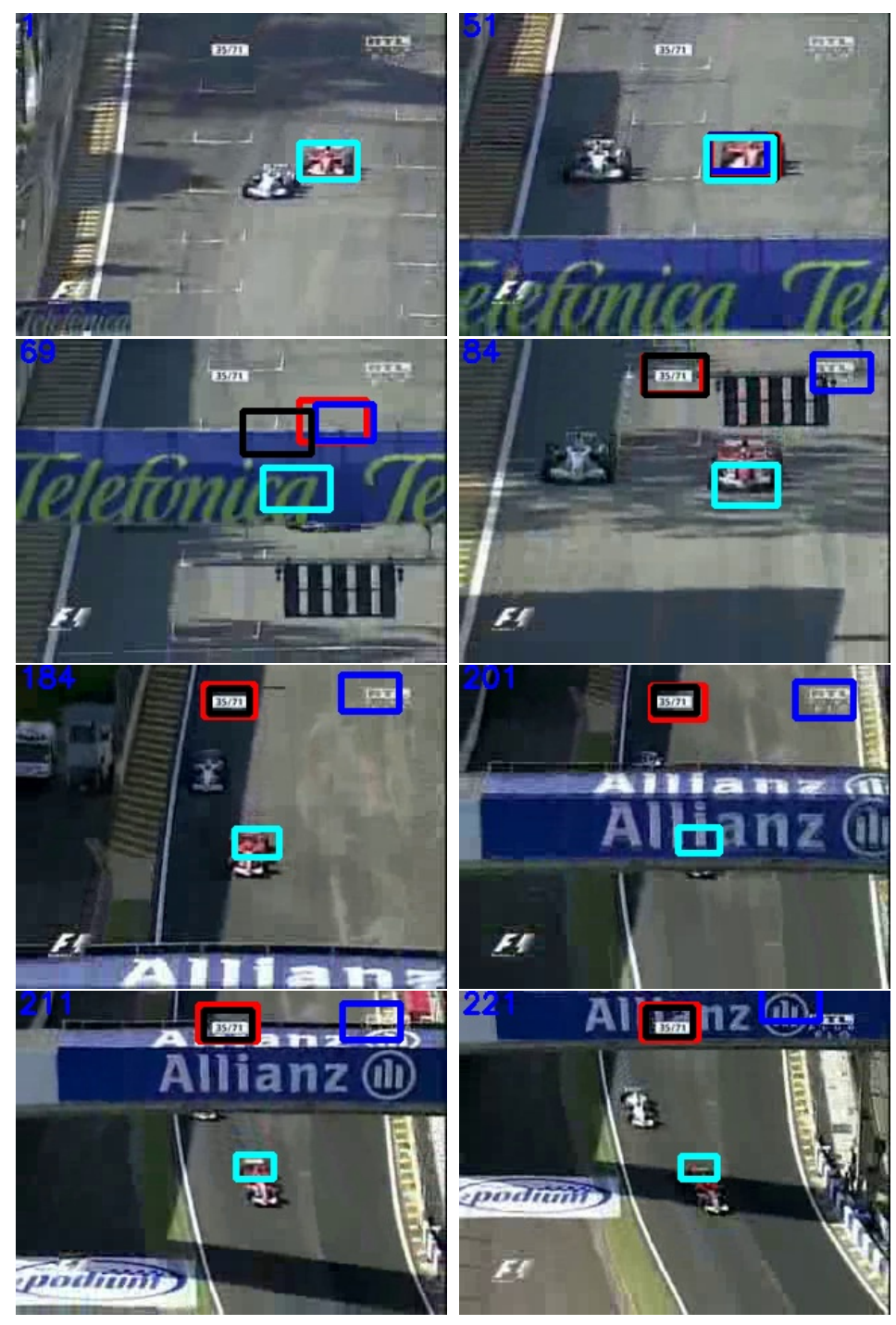

Figure 12: Qualitative experiment on target tracking through occlusions. Cyan: SVK tracker, red: MS tracker, blue: Kalman-MS tracker, black: IMM-MS tracker. 


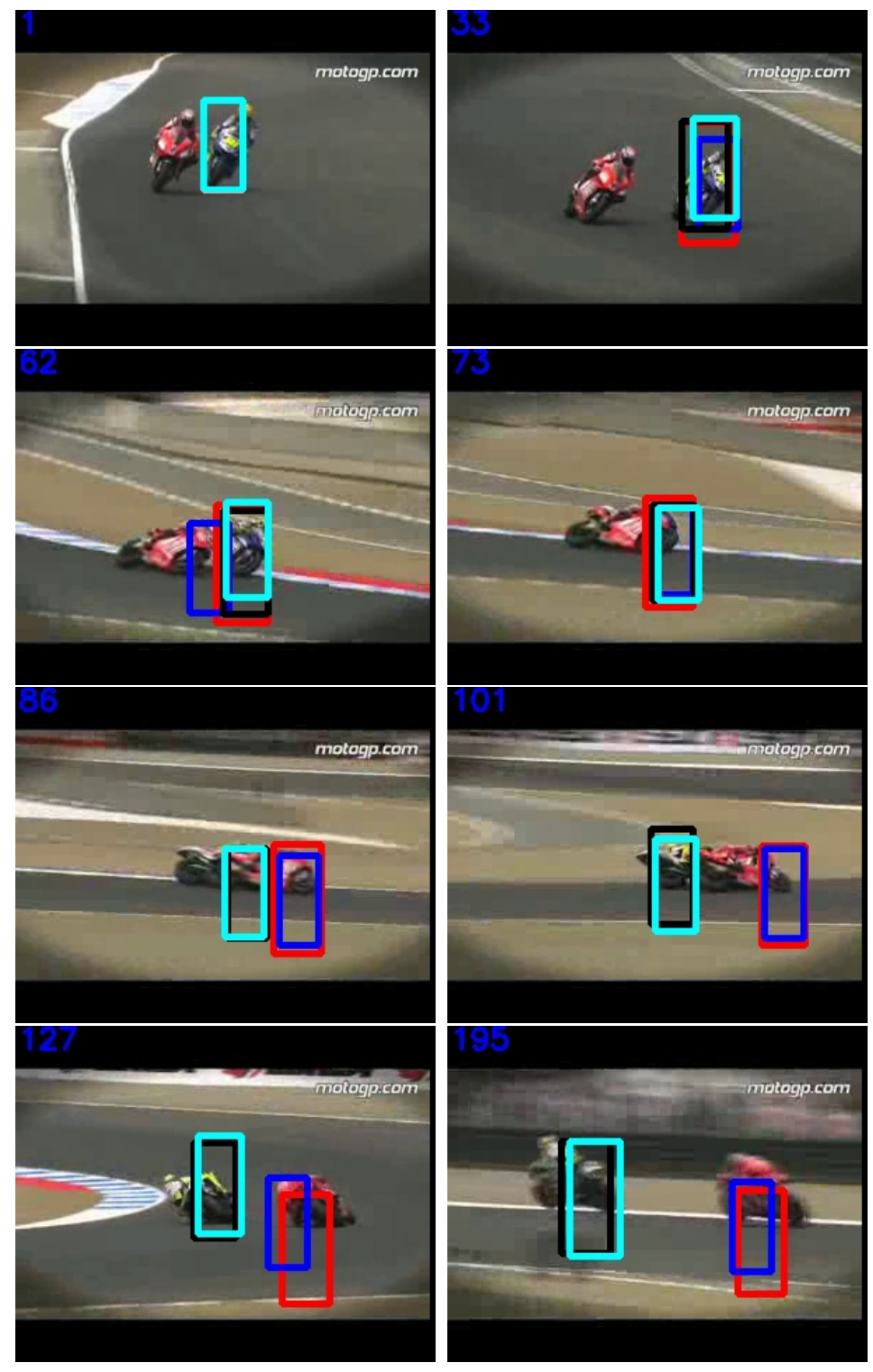

Figure 13: Qualitative experiment on target tracking through occlusions. Cyan: SVK tracker, red: MS tracker, blue: Kalman-MS tracker, black: IMM-MS tracker. 

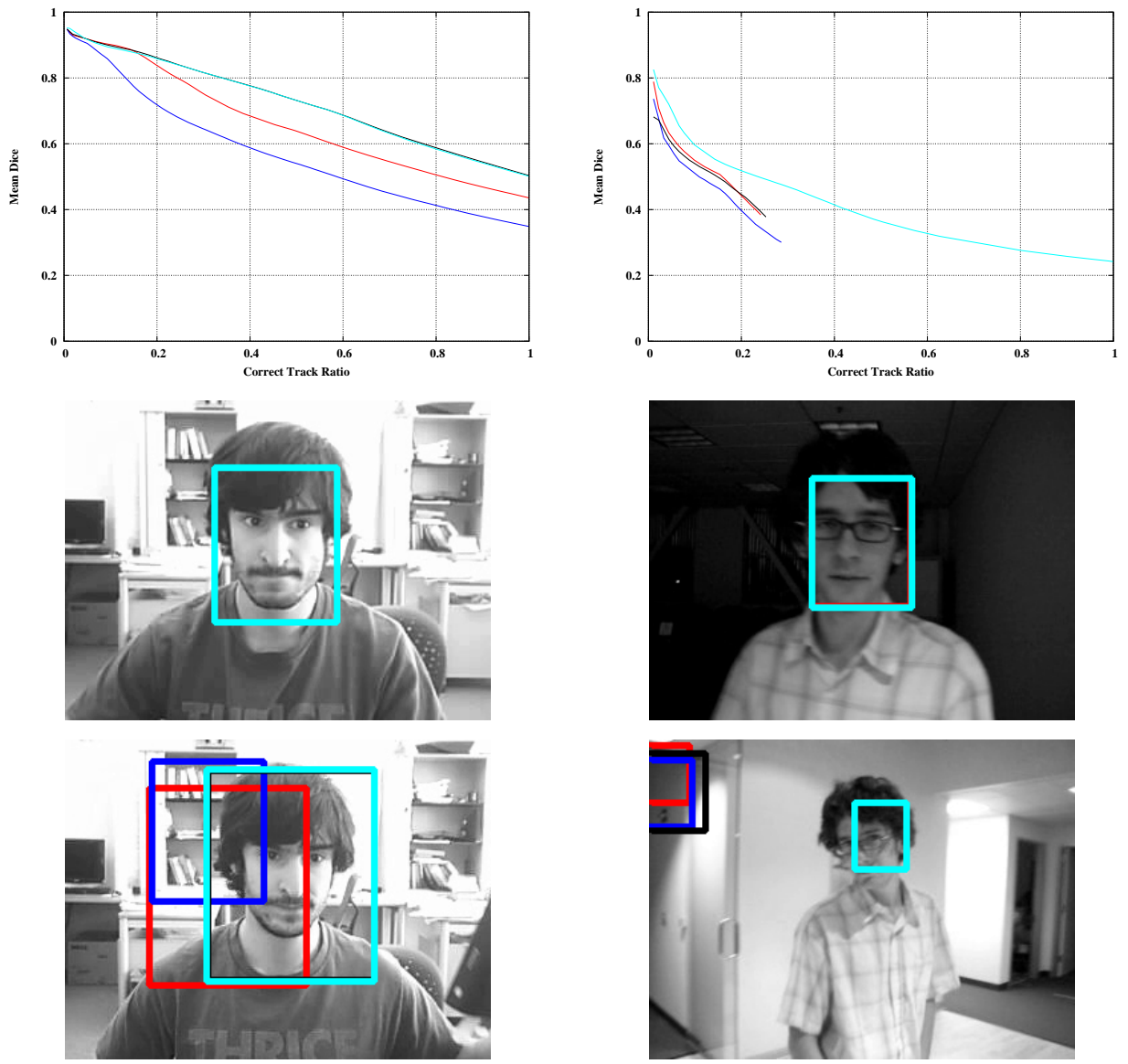

(a)

(b)

Figure 14: Quantitative results. From top to bottom: quantitative results, first frame, exemplar frame from the sequence. (a) Faceocc2 sequence; (b) DavidIndoor sequence. 

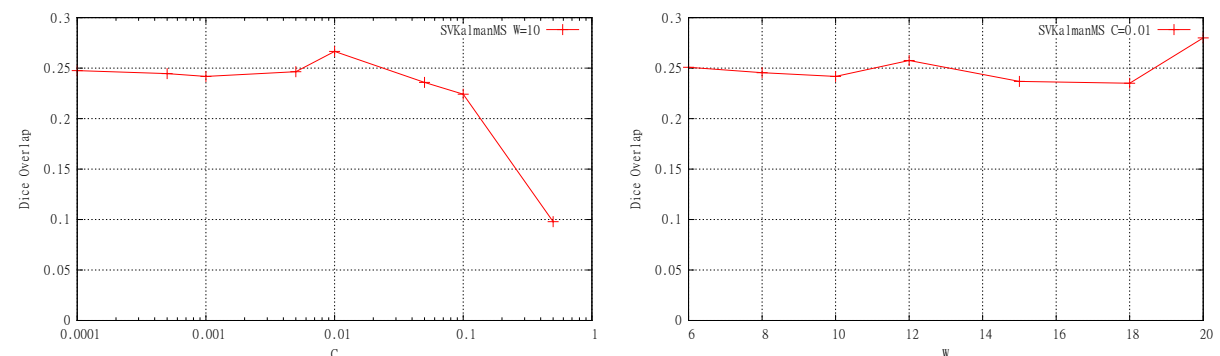

Figure 15: Sensitivity of the proposed algorithm with respect to parameters value. 